\title{
Observation of a novel reduced-turbulence regime with boron powder injection in a stellarator
}

Federico Nespoli ( $\nabla$ fnespoli@pppl.gov )

Princeton Plasma Physics Laboratory https://orcid.org/0000-0001-7644-751X

\section{Suguru Masuzaki}

National Institute for Fusion Science https://orcid.org/0000-0003-0161-0938

Kenji Tanaka

National Institute for Fusion Science

\section{Naoko Ashikawa}

National Institute for Fusion Science

Mamoru Shoji

National Institute for Fusion Science

Erik Gilson

Princeton Plasma Physics Laboratory

Robert Lunsford

Princeton Plasma Physics Laboratory

\section{Tetsutaro Oishi}

National Institute for Fusion Science

Katsumi Ida

National Institute for Fusion Science

\section{Mikirou Yoshinuma}

National Institute for Fusion Science

\section{Yuki Takemura}

National Institute for Fusion Science

\section{Toshiki Kinoshita}

Kyushu University https://orcid.org/0000-0003-3930-4434

\section{Gen Motojima}

National Institute for Fusion Science

Naoki Kenmochi

National Institute for Fusion Science

Gakushi Kawamura

National Institute for Fusion Science

Chihiro Suzuki

National Institute for Fusion Science

\section{Alex Nagy}


Princeton Plasma Physics Laboratory

\section{Alessandro Bortolon}

Princeton Plasma Physics Laboratory

Novimir Pablant

Princeton Plasma Physics Laboratory

Albert Mollen

Princeton Plasma Physics Laboratory

Naoki Tamura

National Institute for Fusion Science

\section{David Gates}

Princeton University https://orcid.org/0000-0001-5679-3124

\section{Tomohiro Morisaki}

National Institute for Fusion Science

\section{Article}

Keywords: Confinement Regime, Turbulent Fluctuations, Line Averaged Electron Density, Resonant Radio Frequency, Hydrogen and Deuterium Plasmas

Posted Date: June 30th, 2021

DOI: https://doi.org/10.21203/rs.3.rs-614131/v1

License: (c) (i) This work is licensed under a Creative Commons Attribution 4.0 International License. Read Full License

Version of Record: A version of this preprint was published at Nature Physics on January 10th, 2022. See the published version at https://doi.org/10.1038/s41567-021-01460-4. 


\title{
Observation of a novel reduced-turbulence regime with boron powder injection in a stellarator
}

F. Nespoli ${ }^{* 1}$, S. Masuzaki ${ }^{2,3}$, K. Tanaka ${ }^{2,4}$, N. Ashikawa ${ }^{2,3}$, M. Shoji ${ }^{2}$, E.P. Gilson ${ }^{1}$, R. Lunsford ${ }^{1}$, T. Oishi ${ }^{2,3}$, K. Ida ${ }^{2,3}$, M. Yoshinuma ${ }^{2,3}$, Y. Takemura ${ }^{2,3}$, T. Kinoshita ${ }^{4}$, G. Motojima ${ }^{2,3}$, N. Kenmochi ${ }^{2,3}$, G. Kawamura ${ }^{2,3}$, C. Suzuki ${ }^{2}$, A. Nagy ${ }^{1}$, A. Bortolon ${ }^{1}$, N.A. Pablant ${ }^{1}$, A. Mollen ${ }^{1}$, N. Tamura ${ }^{2}$, D.A. Gates ${ }^{1}$, T. Morisaki ${ }^{2,3}$

${ }^{1}$ Princeton Plasma Physics Laboratory, 100 Stellarator Road, Princeton, NJ 08540, United States of America

${ }^{2}$ National Institute for Fusion Science, 322-6 Oroshi-cho Toki, Gifu 509-5292, Japan

${ }^{3}$ The Graduate University for Advanced Studies, SOKENDAI, 322-6 Oroshi-cho Toki, Gifu 509-5292, Japan

${ }^{4}$ Interdisciplinary Graduate School of Engineering Sciences, Kyushu University, Kasuga, Fukuoka, 816-8580, Japan

*Corresponding author. Email: fnespoli@pppl.gov

\begin{abstract}
We report the first observation of a novel confinement regime in a stellarator plasma, characterized by increased confinement and reduced turbulent fluctuations. The transition to this new regime is driven by the injection of sub-millimetric boron powder grains into the plasma. With the line averaged electron density being kept constant, substantial increase of stored energy, electron and ion temperature have been observed. At the same time, the amplitude of the plasma turbulent fluctuations is halved. While lower frequency fluctuations are damped, higher frequency modes in the range $100 \leq f[k H z] \leq 200$ are excited. The access to this regime has been observed for different heating schemes, namely with both electron and ion cyclotron resonant radio frequency, and neutral beams, for both directions of the magnetic field, and for both hydrogen and deuterium plasmas.
\end{abstract}

\section{Introduction}

Stellarators are one of the most promising concepts for magnetic confined nuclear fusion, which could provide a clean alternative to fossil fuels and nuclear fission for mass energy production. Unlike tokamaks, their 3D magnetic filed is provided entirely by external coils, removing the need for a current to flow into the plasma, which makes it prone to instabilities and violent disruptions. This also allows the magnetic field to be tailored to minimize neoclassical transport and improve confinement. At present, the biggest degradation of confinement is given by plasma turbulence, resulting in an increased "anomalous" transport. While in principle possible, optimizing the stellarator magnetic field to reduce turbulent transport is extremely challenging due to the huge computational cost of 3D turbulence simulations. It is therefore fundamental to reduce turbulence in order to maximise the plasma confinement, finally determining the amount of fusion reactions.

In this article, we report the observation of a novel confinement regime in the Large Helical Device (LHD) stellarator [1], characterized by widespread reduction of turbulence across the plasma cross-section. In this regime, the line-averaged density remains unvaried, and both ion and electron temperature increase remarkably, without ELM-like bursts typical of H-mode [2]. The transition to this new regime, observed at constant heating power and for different heating sources, is triggered by the injection of sub-millimetric boron (B) powder into the plasma. 


\section{Motivation}

The impurity powder dropper (IPD) is a device for injecting controlled amounts of sub-millimeter powder grains into the plasma under the action of gravity [3]. Its main applications is to perform a real-time boronization by injecting $\mathrm{B}$ powder (and $\mathrm{B}$ composites such as $\mathrm{BN}$ and $\mathrm{B}_{4} \mathrm{C}$ ) into the plasma. The powder, penetrating into the hot plasma, evaporates, and the resulting $\mathrm{B}$ ions are eventually deposited on the plasma facing surfaces creating a thin boron layer. Advantages over the standard glow discharge boronization include no need for the toxic diborane gas $B_{2} H_{6}$, and to interrupt the plasma operation. This technique has been shown to improve wall conditioning already in tokamaks $[4,5,6,7]$, reducing wall recycling and impurity content, and in general increasing the plasma performances by accessing lower plasma collisionalities. Furthermore, the IPD has revealed itself an effective tool for ELMs suppression, allowing the access ELM-free H-mode [8]. A similar powder injection technique has been recently employed on the W7-X stellarator, showing an improvement of confinement [9], most likely induced by the modification of the plasma profiles and in a change of the radial electric field.

The IPD has recently been installed on LHD, with the final goals of improving the plasma performances, and assessing the viability of the real time boronization technique in steady-state operation. Indeed, LHD is capable of extremely long discharges, up to one hour. The installation of the IPD on LHD has been guided by predictive simulations with the coupled EMC3-EIRENE and DUSTT codes [10], maximising the penetration of the powder into the plasma. The successful injection of B and BN powder in the unique LHD plasma configuration, featuring a double-null like cross section with predominantly poloidal magnetic field in the divertor, coupled to the confined plasma by a thick ergodic layer, has been demonstrated in 4-seconds-long plasmas [11], for a wide range of mass injection rates, plasma density, and heating power.

\section{Results}

A new set of powder injection experiments has been performed on LHD, featuring longer plasma duration, in the "inward shifted" magnetic configuration (position of the magnetic axis $R_{a x}=3.6$ $\mathrm{m})$. B powder grains with diameter $d=150 \mu \mathrm{m}$ have been injected for a duration $t_{d} \geq 5 \mathrm{~s}$ in plasmas with different heating sources. A few seconds into powder injection, the plasma performance is observed to improve, with marked increase of both electron and ion temperature $\left(T_{e}, T_{i}\right)$ and plasma stored energy $W_{p}$. Four different examples are shown in Fig. 1, corresponding to different heating schemes: both electron and ion cyclotron resonant heating $(\mathrm{ECH}, \mathrm{ICH})$ for subplot a, ECH only in b (but with perpendicular diagnostic neutral beam (pNB) for charge exchange spectroscopy $(\mathrm{CXS})), \sim 3.5 \mathrm{MW}$ of NB in $\mathrm{c}$, and $\sim 6 \mathrm{MW}$ of NB in $\mathrm{d}$. The magnetic field direction for case $\mathrm{d}$ is reversed with respect to cases a-c. The main plasma ion is deuterium (D) in cases a and b, while it is hydrogen $(\mathrm{H})$ in cases $\mathrm{c}$ and $\mathrm{d}$. For all cases, the line averaged density is comprised in the range $2.7 \leq n_{e, a v}\left[10^{19} m^{-3}\right] \leq 3.7$. The relative increase of $T_{e}, T_{i}$ and $W_{p}$ in between before and during B powder injection is, on average, $\left\langle\Delta T_{e} / T_{e}\right\rangle=27 \%,\left\langle\Delta T_{i} / T_{i}\right\rangle=25 \%,\left\langle\Delta W_{p} / W_{p}\right\rangle=17 \%$.

For all cases, a discharge with $\mathrm{B}$ powder injection (red) is compared with a reference discharge without powder (blue). We remark how in cases b-d, the pulsed operation of the diagnostic beam results in periodic variations on most considered quantities. In case $\mathrm{c}$, huge variations in the plasma at $t \sim 5.3 \mathrm{~s}$ and $t \sim 7.2 \mathrm{~s}$ are due to the change of $\mathrm{NB}$, varying from counter-current direction to co-current and vice-versa.

The effect of B powder on the plasma can be better appreciated in case a, where the gas puff is provided in feed-forward. The powder is dropped at $t \sim 22 \mathrm{~s}$, and approximately after $1 \mathrm{~s}$ of free fall 

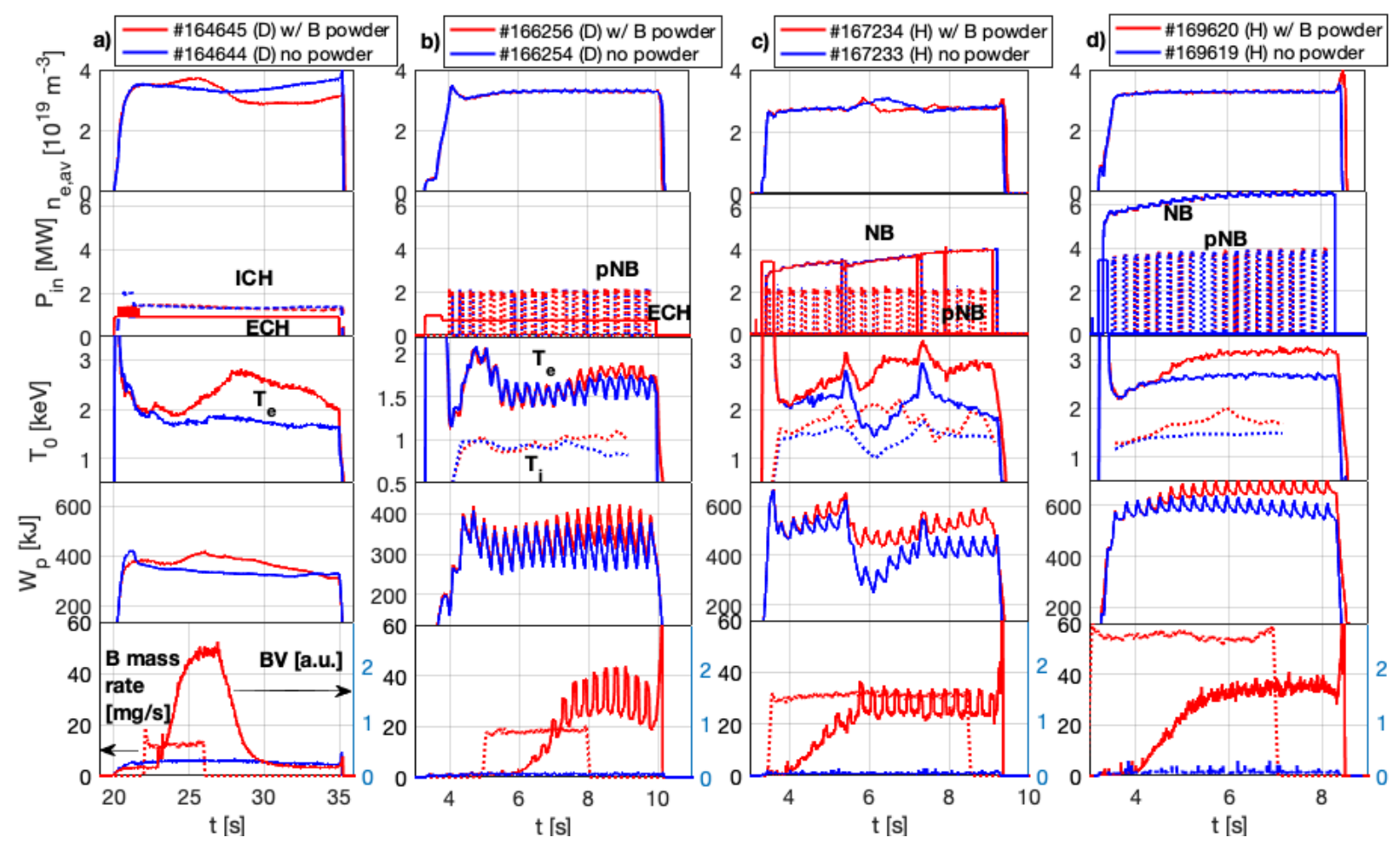

Figure 1: Time traces of, from top to bottom: line averaged density; input power; electron (solid) and ion (dotted) temperature; stored energy; injected boron mass rate (dotted, left axis) and BV spectroscopic line (solid, right axis). Red color for shots with B powder injection with IPD, blue for reference shots (no IPD). Main plasma ion is deuterium for cases a,b and hydrogen for c,d. The magnetic field direction for $\mathrm{d}$ is reversed with respect to a-c.

it enters the plasma, where it is heated and evaporates. The effective injection and vaporization of the powder is confirmed by the sharp increase in the BV line measured by ultraviolet spectroscopy [12]. Due to the extra electron source, the line-averaged electron density $n_{e, a v}$ starts to increase. After a few seconds though, $n_{e, a v}$ decreases below the reference level: this is an effect of the real-time wall conditioning provided by the deposited boron, effectively reducing the recycling at the wall. In the other cases (b-d) the gas puff is operated with a feedback to keep the line averaged density constant, and this effect is masked.

Even though $n_{e, a v}$ remains unvaried, the powder injection changes the shape of the electron density $n_{e}$ profile, as it is shown for one of the previously discussed shots in Fig. 2. During powder injection, $n_{e}$ is increased in the edge region $0.7<r_{e f f} / a_{99}<1$, while $n_{e}$ is slightly decreased for $r_{e f f} / a_{99}<0.7$, rendering the profile more hollow. Here, $r_{e f f}$ the effective minor radius, and $a_{99}$ the minor radius of the flux surface enclosing $99 \%$ of the stored energy. Correspondingly, the slope of the electron temperature profile $T_{e}$ is more strongly increased in the edge region. A similar change is observed for the profiles of ion temperature $T_{i}$ as well. The increase of $n_{e}$ in the edge region is consistent with the powder being vaporized around the LCFS, as it results from coupled EMC3-EIRENE [13] and DUSTT [14] simulations (Fig. 2d). Indeed the powder particles, initially dropped vertically, are deflected by the plasma flow in the divertor leg; nevertheless, they reach the main plasma where they are completely evaporated, depositing neutral B atoms in the range $1 \leq r_{e f f} / a_{99} \leq 1.06$, as shown in Fig. 2e, where the B neutral source is remapped on the normalized radial coordinate $r_{e f f} / a_{99}$ for all the discussed cases. In addition to the extra electron 

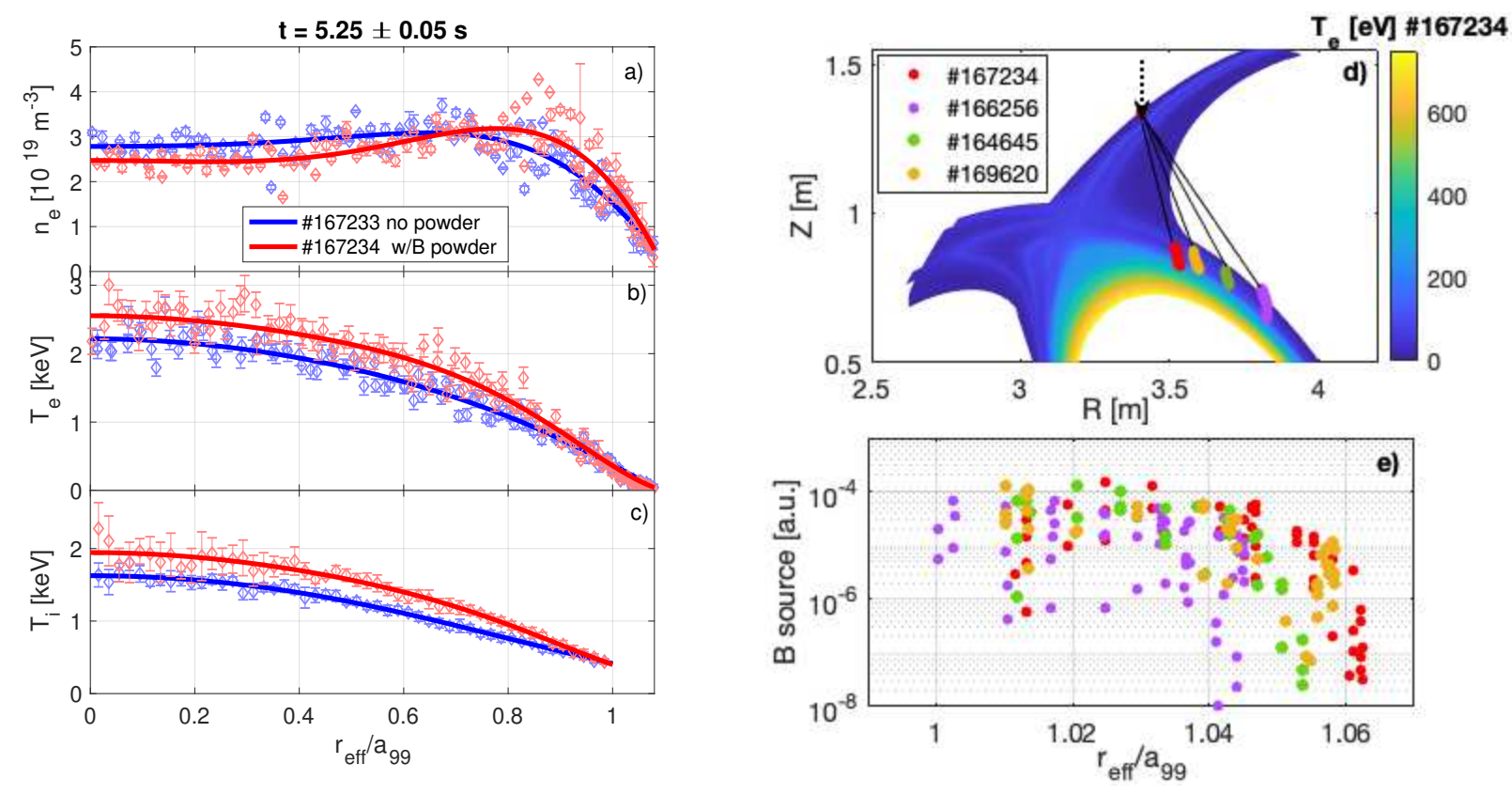

Figure 2: Measured radial profiles of a) electron density $n_{e}$ b) electron temperature $T_{e} \mathrm{c}$ ) ion temperature $T_{i}$, averaged over a $100 \mathrm{~ms}$ time window, in the case with (red) and without (blue) B powder injection. Polynomial fits of the profiles are shown with solid lines. d) $T_{e}$ from EMC3EIRENE simulations for one case (\#167234), together with a B powder grain trajectories (black) computed by the DUSTT code, with the resulting B neutral atom source(dots). e) B neutral source remapped on the normalized radial coordinate $r_{e f f} / a_{99}$.

source provided by the powder, the created B ions are deposited on the plasma facing surfaces, reducing hydrogen recycling and impurity influx. This results in a two-fold reduction of $n_{e}$ in the divertor, as measured by embedded Langmuir probe arrays. The time evolution of the peak value of $n_{e}$ at the strike point is plotted in black in Fig. 3c for a shot with powder injection (solid line) and for the reference discharge (dotted line). $H_{\alpha}$ radiation is also decreased (red traces), suggesting a reduction of recycling. Another indication of the effective real-time boronization is the reduction of impurity influx from the plasma facing components (Fig. 3d), as suggested from the decrease in CVI (red) and FeXVI (blue) radiation lines. The decrease in $\mathrm{C}$ concentration is also confirmed by CXS measurements. The above mentioned increase of $n_{e}$ close to the LCFS due to the powder injection, combined with the decrease of $n_{e}$ at the divertor, causes the density profile to steepen in the plasma edge, as shown in Fig. 3a, where the difference in normalized gradient $\left(d n_{e} / d \rho\right) / n_{e}$ in between the powder injection shot and the no-powder reference is plotted, with $\rho=r_{e f f} / a_{99}$. The steepening of the gradient appears to originate around $r_{e f f} / a_{99} \geq 1$ and propagate inwards, together with an increase of the electron temperature gradient (Fig. 3b).

Simultaneously, the turbulent fluctuation level in the confined plasma is observed to be reduced to approximately half its value before powder injection, as it is shown in Fig. 3e, displaying the amplitude of the the density fluctuations measured by 2D phase contrast imaging (PCI) $[15,16]$, averaged over the whole plasma cross section.

The radially resolved profiles of the density fluctuation amplitude and their velocity in the direction perpendicular to the field line $v_{\perp}$ are plotted in Fig. 4a,b, before (dashed lines) and during B powder injection (solid lines). A discharge with $\mathrm{B}$ powder injection (red) is compared with a 


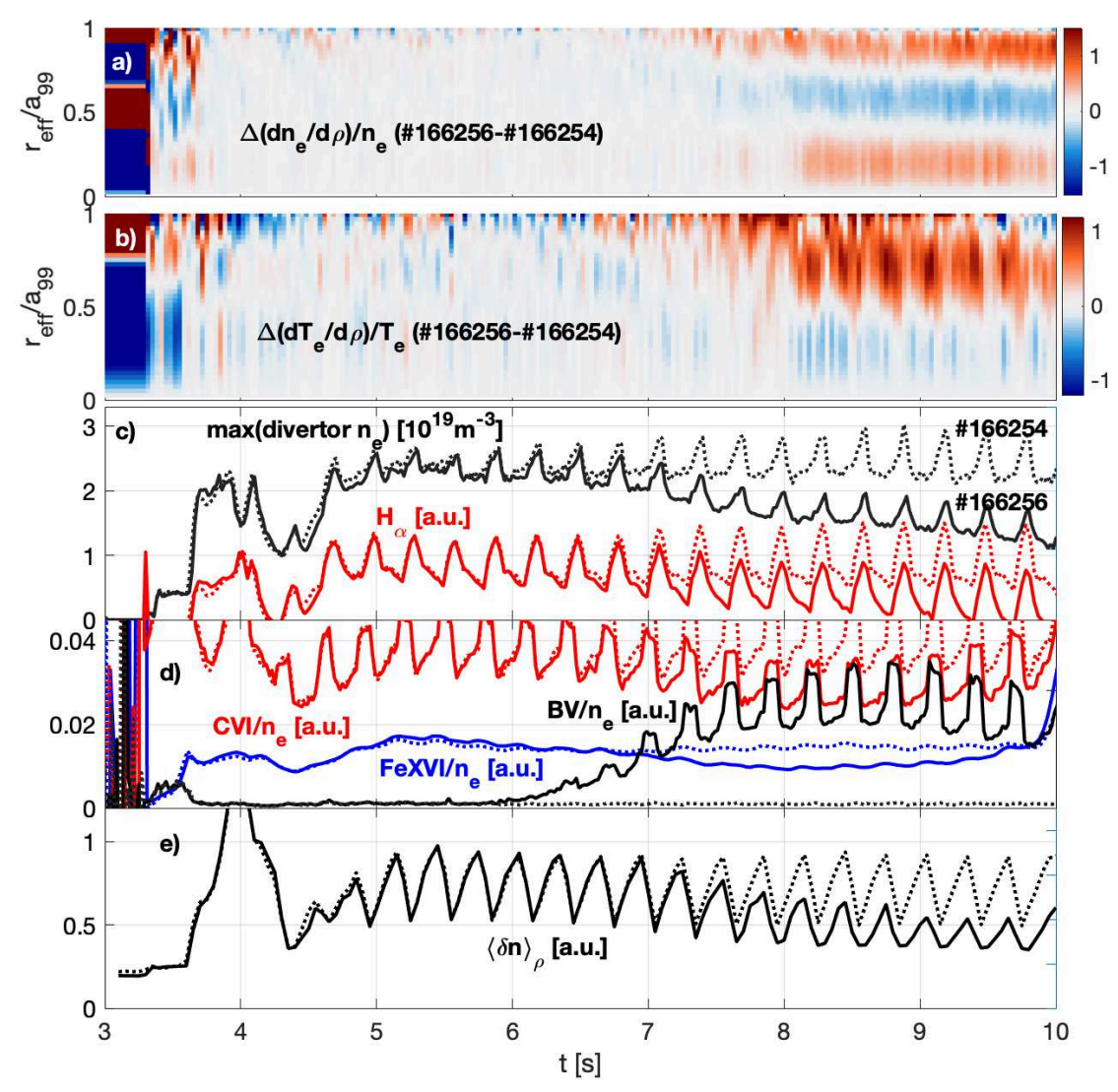

Figure 3: Time evolution of difference of normalized gradient between a powder injection shot (\#166256) and its reference (\#166254) for electron density (a) and temperature (b). c) Peak value of divertor density measured by Langmuir probes (black) and $H_{\alpha}$ radiation (red). d) Evolution of spectroscopic lines CVI (red), FeXVI (blue) and BV (black). e) Average value of the density fluctuation amplitude measured by PCI. Solid lines are for the powder injection shot \#166256, and dotted lines for the reference \#166254. Periodic oscillations at $3.33 \mathrm{~Hz}$ are due to the pulsed operation of diagnostic NB.

reference shot (blue). The shaded area accounts for the variation in time over a time window of $0.4 \mathrm{~s}$, to avoid instantaneous variations caused by the pulsed diagnostic NB. As the turbulent fluctuation amplitude is substantially reduced across the whole cross section (no measurements are available for $\left|r_{e f f} / a_{99}\right|<0.37$ ), their velocity $v_{\perp}$ is doubled in the edge of the plasma $\left(v_{\perp}\right.$ is directed in the e-diamagnetic direction, resulting in positive/negative values in the lab frame when measured at the bottom/top of the plasma $\left.r_{e f f} / a_{99} \lessgtr 0\right)$. Radially resolved power spectral density in terms of perpendicular wave number $k_{\perp}$ are shown in Fig. 4c,d before and during B powder injection respectively. Before the powder injection, the PSD peaks for wave-numbers in the range $0.2 \leq k_{\perp}\left[\mathrm{mm}^{-1}\right] \leq 0.4$, consistent with ion temperature gradient (ITG) driven instabilities. In Refs. $[17,18]$, similar PCI measurements were compared with gyrokinetic simulations, determining that the observed fluctuations are indeed due to ITG turbulence. Dedicated gyrokinetic simulations are needed to confirm this result for the cases exposed here, and are foreseen for future works. During B powder injection, these ITG-like fluctuations are substantially suppressed in the confined plasma $r_{e f f} / a_{99}<1$, and in general across the whole spectrum. Conversely, turbulence is slightly enhanced 

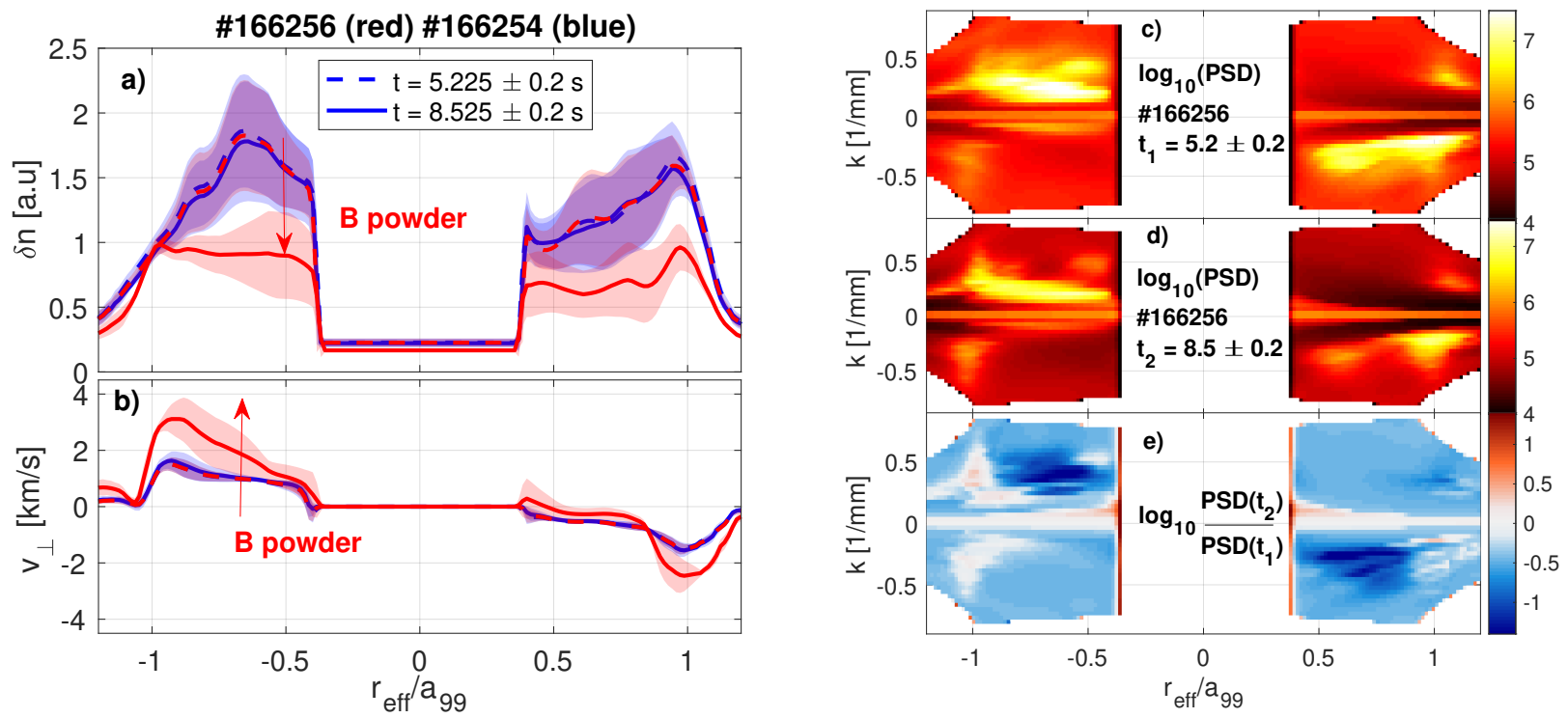

Figure 4: Radial profiles of a) turbulent fluctuation amplitude and b) their perpendicular velocity for a powder injection shot (\#166256, red) and its reference (\#166254, blue), at $t=5.2 \mathrm{~s}$ (before injection, dashed lines) and $t=8.5 \mathrm{~s}$ (during injection, solid lines). Radially resolved power spectral density in terms of wave number for \#166256 before (c) and during (d) powder injection, and their ratio (e).
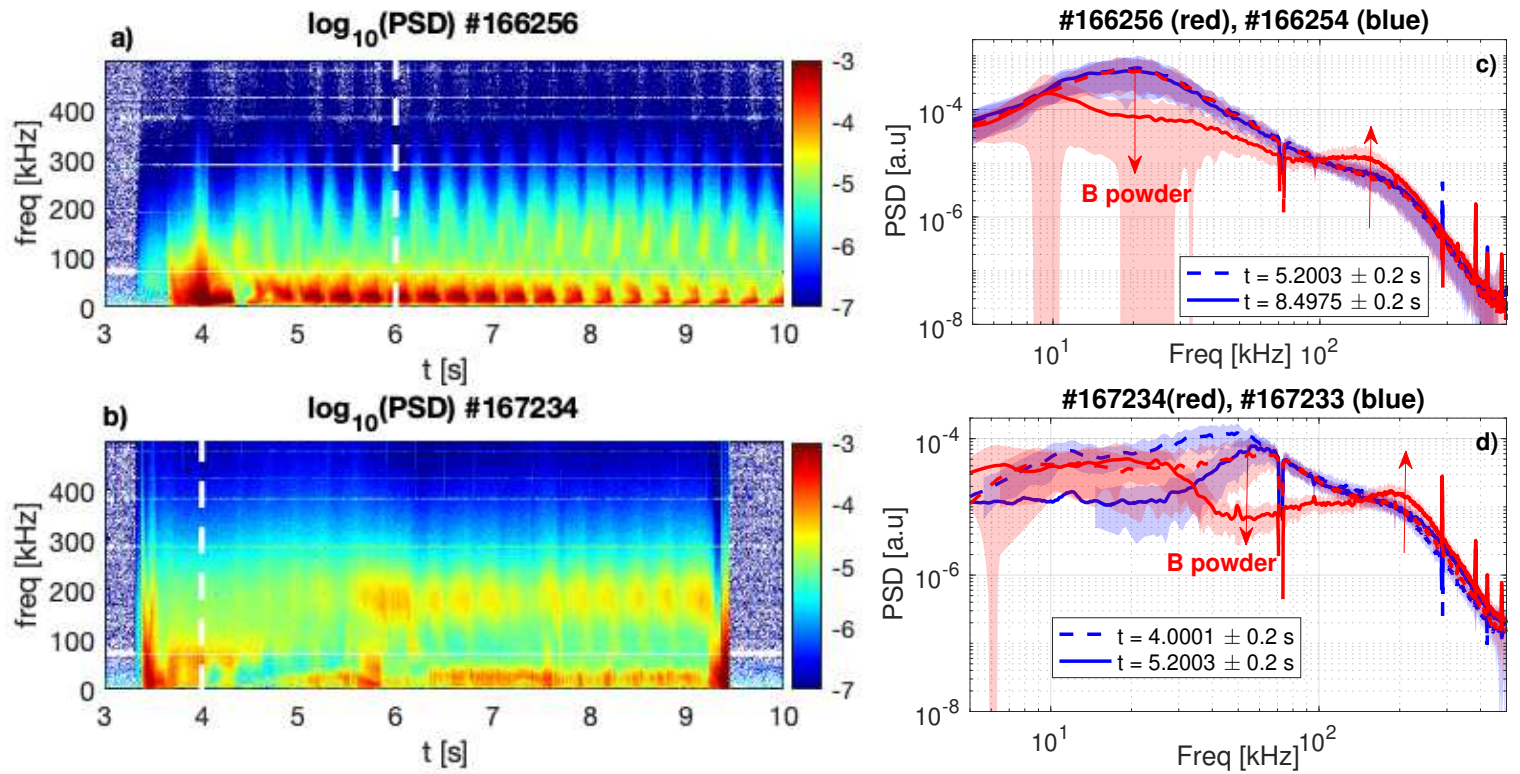

Figure 5: Spectrogram of the line integrated PCI signal for \#166256 (a) and \#167234 (b). The white dashed line indicates the approximate time of the boron powder entering the plasma. c,d) Comparison of time averaged turbulence spectrum for the B injection shot (red) and their reference shot (blue), at time before (dashed lines) and during (solid lines) powder injection. 
around the LCFS $r_{e f f} / a_{99} \sim 1$, as it emerges from Fig. 4e, displaying the logarithm of the ratio of the PSD during $\left(t=t_{2}=8.5 \pm 0.2 \mathrm{~s}\right)$ and before $\left(t=t_{1}=5.2 \pm 0.2 \mathrm{~s}\right)$ B powder injection,

$$
C=\log _{10} \frac{P S D\left(t=t_{2}\right)}{P S D\left(t=t_{1}\right)}
$$

so that $C<0$ and $C>0$ means reduction and increase of turbulence, respectively. Turbulence is therefore reduced on most of the plasma cross section, practically suppressed in correspondence of the peaks before injection $\left(k_{\perp} \sim 0.3 \mathrm{~mm}^{-1}\right)$, and slightly enhanced around the LCFS $\left(r_{e f f} / a_{99} \sim 1\right)$. The additional enhancement for $k_{\perp} \leq 0.1 \mathrm{~mm}^{-1}$ close to the plasma core $r_{\text {eff }} / a_{99} \leq 0.5$ could be an artifact due to insufficient diagnostic resolution for small $k$ values.

This change is reflected in the time evolution of the power spectrum of the line integrated PCI signal (probing all $r_{e f f} / a_{99}>0.4$ ), shown in Fig. 5a,b for shot \#166256 (D, ECH+pNB heated) and \#167234 (H, NB-heated). As powder is injected (white dashed line), the dominant low-frequency fluctuations are suppressed, and a new mode emerges in the range $100 \leq f[k H z] \leq 200$. Time averaged spectra over a window of $0.4 \mathrm{~s}$, in order to attenuate the variations due to the pulsed diagnostic NB, are plotted in Fig. 5c,d for the same shots as in a,b (red) and compared to their reference shot (blue), at times before (dashed lines) and during (solid lines) powder injection. Once again, fluctuations in the range $10 \leq f[k H z] \leq 100$ are damped, while a new peak emerges in the spectrum for $f \sim 100-200 \mathrm{kHz}$. In Ref. [26], a similar difference in PCI spectra has been observed in between isotope mixing and non-mixing discharges. In the first case, the spectrum peaks at $\sim 20$ $\mathrm{kHz}$, identified with ITG turbulence. At lower collisionalities, ITG turbulence is stabilized and trapped electron modes (TEM) are destabilized instead at the edge of the plasma, where gradients are steeper, resulting in a peak at $\sim 80 \mathrm{kHz}$ in the PCI spectrum.

The decrease in turbulence is observed on the whole cross section, except close to the LCFS region $r_{e f f} / a_{99} \sim 1$. Conversely to the case of a H-mode, where turbulence is suppressed in the vicinity
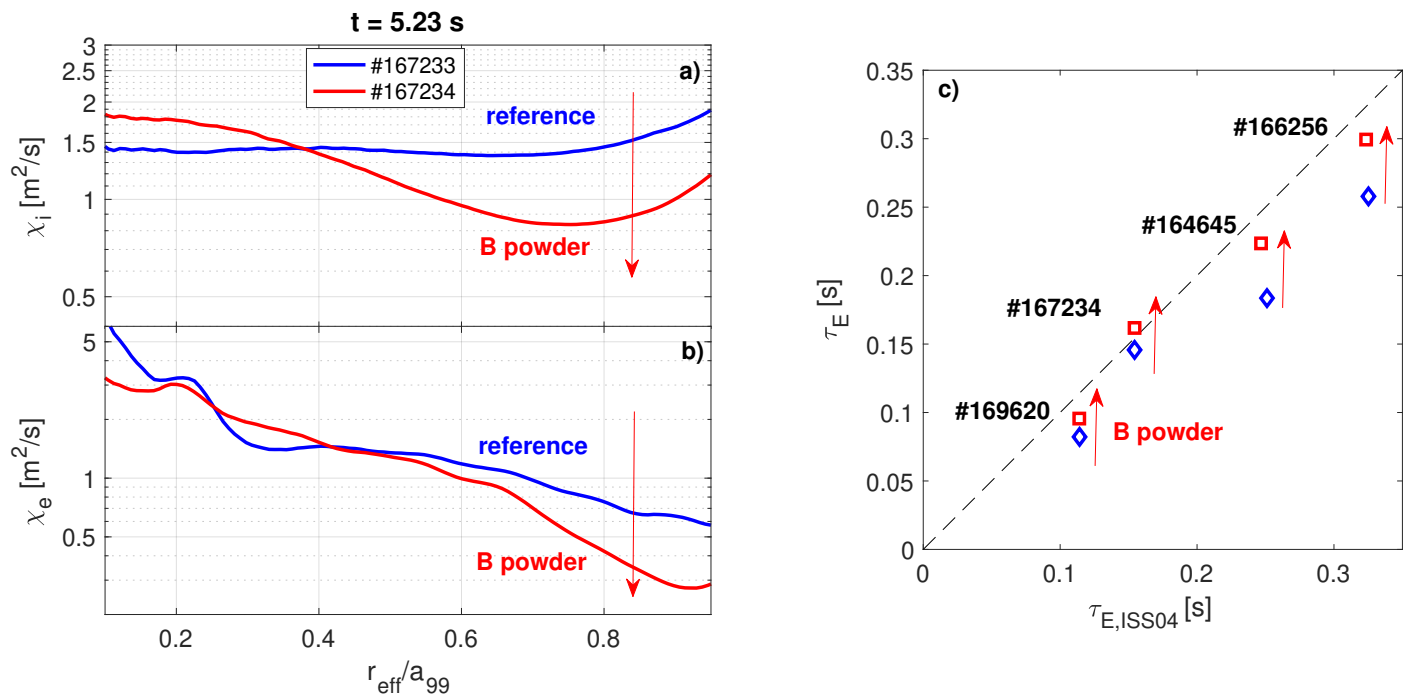

Figure 6: Radial profiles of heat conductivities for ions (a) and electrons (b), comparing a discharge with powder injection (red) with a reference discharge (blue). c) Measured energy confinement time $\tau_{E}$ during B powder injection (red squares), and for the reference shots at the same time (blue diamonds). The experimental $\tau_{E}$ is plotted against the predicted energy confinement time from the international stellarator scaling [28]. 
of the LCFS only, due to a increased shear in poloidal velocity and therefore in the radial electric field $E_{r}$, since the poloidal velocity is mainly given by the $\mathbf{E} \times \mathbf{B}$ drift. In Ref. [9], confinement improvement has been observed in $\mathrm{W} 7-\mathrm{X}$ during $\mathrm{B}_{4} \mathrm{C}$ powder injection, which has been judged to be consistent with a change in $E_{r}$. In our case, no significant change in radial electric field is expected in the phase where the powder is injected into the plasma. The ambipolar radial electric field has been computed using the neoclassical transport code SFINCS [19], for all cases (except \#164645 where no $T_{i}$ measurement is available), comparing plasma with and without powder injection. In all cases, no change in $E_{r}$ that could result in an improvement of confinement emerges from the simulation results. The increase in $T_{e}, T_{i}, W_{p}$ observed during powder injection is associated with a reduced energy transport in the edge of the plasma, and an increase in energy confinement time. The heat conductivities for ions and electrons, $\chi_{i}$ and $\chi_{e}$ respectively, are computed using the DYTRANS module of TASK3D-a [27], performing a dynamics transport analysis. As a result (shown in Fig. 6 for a NBI heated case), both $\chi_{i}$ and $\chi_{e}$ are reduced in the plasma edge during powder injection. $\chi_{i}$ is reduced by up to $40 \%$ for $r_{e f f} / a_{99}>0.4$, while $\chi_{e}$ is reduced by up to $50 \%$ for $r_{e f f} / a_{99}>0.5$. The DYTRANS results are consistent with the reduction of turbulent transport in the edge of the plasma during B powder injection. The measured energy confinement time $\tau_{E}$ (red squares in Fig. 6 , plotted against the value predicted from the international stellarator scaling $\tau_{E, I S S 04}[28]$ ), is also increased during B powder injection, the improvement being in between $17 \%$ and $25 \%$ when compared to the reference shot at the same time (blue diamonds).

\section{Conclusions}

The Impurity Powder Dropper has been used to inject sub-millimetric boron (B) powder into the LHD plasma. During powder injection, the electron and ion temperature $T_{e}, T_{i}$ and the plasma stored energy $W_{p}$ have been observed to increase by approximately $27 \%, 25 \%$ and $17 \%$ respectively. The improvement has been observed for different heating schemes $(\mathrm{ECH}+\mathrm{ICH}, \mathrm{ECH}+\mathrm{pNB}, \mathrm{NBH})$, for both directions of the magnetic field, and for both $\mathrm{H}$ and $\mathrm{D}$ plasmas. At the same time, the turbulent fluctuation amplitude measured by PCI, most possibly due to ITG type of turbulence, is observed to decrease up to a factor 2 , and a secondary peak in the power spectrum of the line integrated signal has been observed to emerge at higher frequencies $f>100 \mathrm{kHz}$. When powder is injected, the density profile is steepened in the edge region and made more hollow in the center, due to an additional electron source for $r_{e f f} / a_{99} \geq 1$ provided by the vaporization of the powder itself, as determined by EMC3-EIRENE and DUSTT simulations. Simultaneously, the B ions deposit on the plasma facing components, changing the wall conditions in real time: the impurity influx from the wall is reduced, together with the recycling at the divertor plates. The latter results in lower plasma density in the divertor region, contributing to increase the density gradient. The modification of the $n_{e}$ profile at the edge is followed by the steepening of $T_{e}$ and $T_{i}$ in the same region, resulting finally in an increase in their value on axis. Accordingly, the analysis of dynamic transport exhibits a reduction of the heat conductivities for electrons and ions $\chi_{e}$ and $\chi_{i}$ in the plasma edge. The measured energy confinement time $\tau_{E}$ is also observed to increase. As the $n_{e}$, $T_{e}$ and $T_{i}$ profiles are changed, the ambipolar radial electric field computed by the SFINCS code remains mainly unvaried. This, together with reduction of the turbulent fluctuations across the whole plasma cross section, are in contrast with an H-mode type increase of confinement, where the turbulent fluctuations are reduced in the vicinity of the separatrix due to an increased $\mathbf{E} \times \mathbf{B}$ shear, and suggest a different underlying mechanism.

While the reason of the observed improvement of confinement is not yet clear, we suspect it to be due to the suppression of ITG turbulence. This might be an effect of the change in the profiles of the 
plasma quantities, in particular of the peaking of $n_{e}$ in the edge, resulting in a more hollow profile in the center of the plasma. Hollow density profiles have been reported to increase ITG stability, reducing turbulence fluctuations $[18,21]$. Another possibility is turbulence being reduced by the increased effective charge $Z_{\text {eff }}$ due to B injection, also referred to as plasma dilution. Indeed, an increase $Z_{\text {eff }}$ has also been reported to have a stabilizing effect on ITG turbulence [22, 23, 24]. More likely, the combination of the two above mentioned effects might occur in our case, similarly to what reported in Ref. [25]. Dedicated gyrokinetic simulations will be necessary to verify those hypotheses, and are planned for future works.

Furthermore, additional experiments are foreseen on LHD, with the aim of better defining the parameter space where this new regime is observed, and to assess whether it is compatible with conditions relevant to future fusion reactors, i.e. with higher power and density plasmas.

\section{Methods}

Evaluation of plasma quantities and profiles

Throughout the paper, the line averaged electron density $n_{e, a v}$ is measured by means of far infrared interferometry (FIR). Radially resolved profiles of electron density and temperature $n_{e}, T_{e}$ are measured by Thomson scattering (TS). Radially resolved profiles of ion temperature $T_{i}$ are measured by charge exchange spectroscopy (CXS). Using magnetic reconstruction from the VMEC code, all profiles are remapped onto the normalized coordinate $\rho=r_{\text {eff }} / a_{99}$, with $r_{\text {eff }}$ the effective minor radius, and $a_{99}$ the minor radius of the flux surface enclosing $99 \%$ of the stored energy. For each time step, the $n_{e}, T_{e}, T_{i}$ profiles are fitted with polynomials including only even power terms, ensuring zero derivative at $r_{e f f} / a_{99}=0$. This provides smooth spatial profiles and gradients. The values of temperatures on axis (Fig. 1) correspond to the fitted profiles evaluated at $r_{\text {eff }} / a_{99}=0$. For discharges \#164644 and \#164645 in Fig. 1A, the diagnostic neutral beam was not operated and no $T_{i}$ measurement from CXS is available.

Powder injection and evaluation of mass rate

The impurity powder dropper features four independent feeders, composed of a powder reservoir and a vibrating tray. The latter is vibrated by piezoelectric blades through a driving voltage, allowing to control the amplitude of the vibrations and finally the amount of powder delivered to the plasma. Each independent feeder includes an accelerometer, measuring the vibration of the tray actuated by piezoelectric blades, finally determining the injection rate of the powder into the plasma. Calibration curves have been acquired in the laboratory, allowing to convert the amplitude of the accelerometer signal into injected mass rates.

\section{Simulations with EMC3-EIRENE and DUSTT}

Interpretative simulations are performed with the coupled EMC3-EIRENE [13] and DUSTT [14] codes, after Ref. [10]. EMC3 is a fully 3D Monte Carlo code modelling the plasma transport in the edge and scrape-off layer, and it is coupled to EIRENE, describing the neutrals dynamics. The diffusion and thermal conductivities coefficients are set by matching the experimental profiles of plasma density and ion and electron temperatures. Electron density and temperature are measured by Thomson scattering, while the ion temperature is measured by charge exchange spectroscopy. The 3D plasma solutions from the EMC3-EIRENE simulations are then used as the background for the DUSTT simulations, computing the trajectory of a powder grain injected into the plasma. For those simulations, the powder grains are injected vertically at the actual IPD location with an initial velocity of $5 \mathrm{~m} / \mathrm{s}$ directed downwards, consistent with the free fall of the powder grains prior 
to entering the plasma. The powder material is $\mathrm{B}$, and the size of the modelled powder grain is 150 $\mu \mathrm{m}$, matching the ones used in the experiments. As the powder grains enter the plasma, they are progressively heated up to evaporating temperature, providing a localized source of neutral B atoms.

Measurement of turbulent fluctuations

In this work, the turbulent fluctuations characteristics are measured by means of two-dimensional Phase Contrast Imaging (PCI). The diagnostic system and the spectral analysis technique are detailed in Refs. [15, 16]. The amplitude of the density fluctuation is computed from the power spectrum integrated over $\omega$ and $k$ as $\sqrt{\tilde{n}^{2}}$. The cutoff wave-number of the PCI system has been investigated in detail in Ref. [29], showing how fluctuations with wave-number in the range $0.1 \leq k\left[\mathrm{~mm}^{-1}\right] \leq 0.8$ are measured, with an attenuated contributions to the final spectrum for $k<0.2 \mathrm{~mm}^{-1}$.

Energy confinement time

The confinement time is computed from the time trace of the plasma stored energy $W_{p}$, smoothed in time to average over the variations provided by the perpendicular NBI. For ECH, all port-through power is assumed to be absorbed by the plasma. For ICH, it is assumed that $75 \%$ of the input power is absorbed. For NBIs, the absorbed power is assumed to be $P_{a b}=P_{i n}\left[1-\exp \left(-\sigma n_{e, a v} l\right)\right]$, where $\sigma=0.43$ for $\mathrm{H}$ plasmas and $l=1.86 \mathrm{~m}[30]$.

\section{Acknowledgments}

The authors wish to thank the LHD experiment group for the excellent support of this work, and Drs. R. Seki and M. Yokoyama (NIFS) for executing TASK3D-a suite, allowing to conduct transport analyses. This work was conducted within the framework of the NIFS/PPPL International Collaboration, and it is supported by the U.S. DOE under Contract No. DE-AC02-09CH11466 with Princeton University.

\section{Author Contributions}

N.A., E.P.G, R.L., S.M., M.S, F.N., A.N. set up and performed the experiments. T.O., K.I., M.Y., Y.T., K.T., T.K., C.S. set up and operated the diagnostics used in the experiments and ran preliminary analysis. F.N., M.S., G.K., G.M., N.K., N.A.P, A.M. performed numerical modeling of the experiments. F.N., S.M., K.T., K.I, A.B., D.A.G further analyzed and interpreted the data. F.N. prepared the figures and wrote the manuscript. D.A.G. and T.M. supervised the project. All authors reviewed the manuscript and contributed to discussions.

\section{References}

[1] A. Iiyoshi et al., Overview of the Large Helical Device project, Nuclear Fusion 39 (1999) pp.1245-1256.

[2] F. Wagner, A quarter-century of H-mode studies, Plasma Physics and Controlled Fusion 49 (2007)

[3] A. Nagy et al., A multi-species powder dropper for magnetic fusion applications, Review of Scientific Instruments 89 (2018), 10K121 
[4] A. Bortolon et al., Real-time wall conditioning by controlled injection of boron and boron nitride powder in full tungsten wall ASDEX Upgrade, Nuclear Materials and Energy 19 (2019) 384-389

[5] R. Lunsford et al., Active conditioning of ASDEX Upgrade tungsten plasma-facing components and discharge enhancement through boron and boron nitride particulate injection, Nuclear Fusion 59 (2019) 126034

[6] A. Bortolon et al., Observations of wall conditioning by means of boron powder injection in DIII D H-mode plasmas, Nuclear Fusion 60 (2020) 126010

[7] E. P. Gilson et al., Wall Conditioning with Boron Nitride and Boron Powder Injection in KSTAR, submitted to Nuclear Materials and Energy (2021)

[8] Z. Sun et al., Suppression of edge localized modes with real-time boron injection using the tungsten divertor in EAST, Nuclear Fusion 61 (2021) 014002

[9] R. Lunsford et al., Characterization of injection and confinement improvement through impurity induced profile modifications on the Wendelstein 7-X stellarator, submitted to Physics of Plasmas (2021)

[10] M. Shoji et al., Full-torus impurity transport simulation for optimizing plasma discharge operation using a multi-species impurity powder dropper in the large helical device, Contributions to Plasma Physics (2019) e201900101. https://doi.org/10.1002/ctpp.201900101

[11] F. Nespoli et al., First impurity powder injection experiment in LHD, Nuclear Materials and Energy 25 (2020) 100842

[12] T. Oishi et al., Line identification of boron and nitrogen emissions in EUV and VUV wavelength ranges in the impurity powder dropping experiments of LHD and its application to spectroscopic diagnostics, Plasma Science and Technology (2021) https://doi.org/10.1088/2058$6272 / \operatorname{abfd} 88$

[13] Y. Feng et al., Fluid features of the stochastic layer transport in LHD, Nuclear Fusion 48 (2008) 024012

[14] R.D. Smirnov et al., Plasma Physics and Controlled Fusion 49 (2007) 347-371

[15] K. Tanaka et al., Two-dimensional phase contrast imaging for local turbulence measurements in large helical device (invited) Review of Scientific Instruments 79, 10E702 (2008)

[16] C. A. Michael, K. Tanaka, L. Vyacheslavov, A. Sanin, and K. Kawahata, Two-dimensional wave-number spectral analysis techniques for phase contrast imaging turbulence imaging data on large helical device, Review of Scientific Instruments 86, 093503 (2015)

[17] M. Nunami et al., Linear Gyrokinetic Analyses of ITG Modes and Zonal Flows in LHD with High Ion Temperature, Plasma and Fusion Research 6 (2011) 1403001

[18] K. Tanaka et al., Extended investigations of isotope effects on ECRH plasma in LHD, Plasma Physics and Controlled Fusion 62 (2020) 024006

[19] M. Landreman, et al., Comparison of particle trajectories and collision operators for collisional transport in nonaxisymmetric plasmas, Phys. Plasmas 21 (2014) 042503. 
[20] M. Yokoyama et al., Extended capability of the integrated transport analysis suite, TASK3Da, for LHD experiment, Nuclear Fusion 57 (2017) 126016

[21] M. Nakata et al., Gyrokinetic microinstability analysis of high-Ti and high-Te isotope plasmas in Large Helical Device, Plasma Phys. Control. Fusion 61 (2019) 014016

[22] D. R. Mikkelsen et al., Quasilinear carbon transport in an impurity hole plasma in LHD, Physics of Plasmas 21, 082302 (2014)

[23] P. Ennever et al., The effects of dilution on turbulence and transport in C-Mod ohmic plasmas and comparisons with gyrokinetic simulations Physics of Plasmas 22, 072507 (2015)

[24] P. Ennever et al., The effects of main-ion dilution on turbulence in low q95 C-Mod ohmic plasmas, and comparisons with nonlinear GYRO, Phys. Plasmas 23, 082509 (2016)

[25] T. Kinoshita et al., submitted to Plasma Physics and Controlled Fusion (2021)

[26] K. Ida et al., Characteristics of plasma parameters and turbulence in the isotope-mixing and the non-mixing states in hydrogen-deuterium mixture plasmas in the large helical device, Nucl. Fusion 61 (2021) 016012

[27] M. Yokoyama et al., Extended capability of the integrated transport analysis suite, TASK3Da, for LHD experiment, Nucl. Fusion 57 (2017) 126016

[28] H. Yamada et al., Characterization of energy confinement in net-current free plasmas using the extended International Stellarator Database, Nuclear Fusion 45 (2005) 1684-1693

[29] T. Kinoshita et al., Determination of absolute turbulence amplitude by CO2 laser phase contrast imaging, JINST 15 C01045 (2020)

[30] Y. Takeiri et al., High-ion temperature experiments with negative-ion-based neutral beam injection heating in large helical device, Nucl. Fusion 45 (2005) 565-573. 